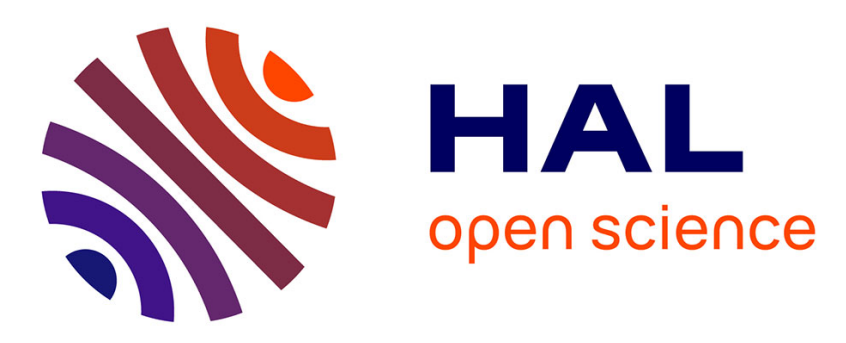

\title{
On the modeling of contact/impact problems between rubber materials
}

\author{
Zhi-Qiang Feng, Qichang He, Benoit Magnain, Jean-Michel Cros
}

\section{To cite this version:}

Zhi-Qiang Feng, Qichang He, Benoit Magnain, Jean-Michel Cros. On the modeling of contact/impact problems between rubber materials. Lecture Notes in Applied and Computational Mechanics, 2006, 2006 (27), pp.87-94. 10.1007/3-540-31761-9_10 . hal-01179017

\section{HAL Id: hal-01179017 \\ https://hal.science/hal-01179017}

Submitted on 28 Jun 2018

HAL is a multi-disciplinary open access archive for the deposit and dissemination of scientific research documents, whether they are published or not. The documents may come from teaching and research institutions in France or abroad, or from public or private research centers.
L'archive ouverte pluridisciplinaire HAL, est destinée au dépôt et à la diffusion de documents scientifiques de niveau recherche, publiés ou non, émanant des établissements d'enseignement et de recherche français ou étrangers, des laboratoires publics ou privés. 


\title{
On the modeling of contact/impact problems between rubber materials
}

\author{
Z.-Q. Feng ${ }^{1}$, Q.-C. $\mathrm{He}^{2}$, B. Magnain ${ }^{1}$ and J.-M. Cros $^{1}$ \\ 1 Laboratoire de Mécanique d'Evry, Université d'Evry, 40 rue du Pelvoux \\ 91020 Evry, France. feng@iup.univ-evry.fr \\ 2 Laboratoire de Mécanique, Université de Marne-la-Vallée, 19 rue A. Nobel \\ 77420 Champs sur Marne, France. he@univ-mlv.fr
}

\begin{abstract}
This work is concerned with the finite element modeling of contact/impact problems between rubber materials. The developed algorithm, namely here Bi-First, combines the bi-potential method for solution of contact problems and the first order algorithm for integration of the time-discretized equation of motion. Numerical examples are given in two cases: multi-contact problem between Blatz-Ko hyperelastic bodies and Love-Laursen's test with a novel hyperelastic model.
\end{abstract}

\section{Introduction}

Problems involving contact and friction are among the most difficult ones in mechanics and at the same time of crucial practical importance in many engineering branches. A large number of algorithms for the modeling of contact problems by the finite element method have been presented in the literature. See for example the monographs by Kikuchi and Oden [1], Zhong [2], Wriggers [3], Laursen [4] and the references therein. De Saxcé and Feng [5] have proposed a bi-potential method, in which an augmented Lagrangian formulation was developed. Feng et al. [6, 7] have successfully applied this method for the modeling of static contact problems between elastic and Blatz-Ko hyperelastic bodies.

For dynamic implicit analysis in structural mechanics, the most commonly used time integration algorithm is the second order algorithm such as Newmark, Wilson, HHT [8]. The first order algorithm has also been proposed by Jean [9] for time stepping in rigid-body dynamic contact problems. Recently, Feng et al. [10] have applied this algorithm for the modeling of impact problems between elastic bodies.

In nonlinear elasticity, there exist many constitutive models to describe the hyperelastic behavior of foam-like or rubber-like materials, such as Blatz-Ko [11], Ogden [12], Gent [13], etc. These models are available in many modern commercial finite element codes. In 1999, Lainé et al. [14] proposed a new 
third order hyperelastic model, namely here the $L V F$ model. The aim of the present paper is to apply the Bi-First algorithm for contact modeling in dynamic cases between rubber materials described by Blatz-Ko and the $L V F$ model. Two numerical examples are performed in this study to show the validity and efficiency of the algorithm developed.

\section{Hyperelastic models}

In the case of hyperelastic laws, there exists an elastic potential function $W$ (or strain energy density function) which is a scale function of one of the strain tensors, whose derivative with respect to a strain component determines the corresponding stress component. This can be expressed by

$$
\mathbf{S}=\frac{\partial W}{\partial \mathbf{E}}=2 \frac{\partial W}{\partial \mathbf{C}}
$$

where $\mathbf{S}$ is the second Piola-Kirchoff stress tensor, $\mathbf{C}$ the right Cauchy-Green deformation tensor and $\mathbf{E}$ the Green-Lagrangian strain tensor. The BlatzKo constitutive law is used to model compressible foam-type polyurethane rubbers [11]. The strain energy density function is given as follows

$$
W=\frac{G}{2}\left(\frac{I_{2}}{I_{3}}+2 \sqrt{I_{3}}-5\right)
$$

where $I_{2}$ and $I_{3}$ are respectively the second and third invariant of $\mathbf{C}$ and $G$ is the shear modulus. Reporting (2) in (1) gives the constitutive relation as follows

$$
\mathbf{S}=G\left[\sqrt{I_{3}}(2 \mathbf{E}+\mathbf{I})^{-1}-(2 \mathbf{E}+\mathbf{I})^{-2}\right]
$$

The $L V F$ constitutive law is proposed by Lainé et al. [14] to describe the isotropic compressible or incompressible rubber-like material. New invariants of $\mathbf{E}:(x, y, z)$ are introduced as follows

$$
x=\sqrt{\operatorname{tr}\left(\mathbf{E}_{d}\right)^{2}} \cos \vartheta, \quad y=\sqrt{\operatorname{tr}\left(\mathbf{E}_{d}\right)^{2}} \sin \vartheta, \quad z=\frac{\operatorname{tr}(\mathbf{E})}{\sqrt{3}}
$$

where $\mathbf{E}_{d}$ is the distortional part of $\mathbf{E}$ and $\vartheta$ the Lode's angle. The strain energy density function of fourth order expressed in terms of the new invariants of the strain tensor is given as follows

$$
\begin{aligned}
W(x, y, z) & =\left(G+\frac{a_{3}}{4} z^{2}\right)\left(x^{2}+y^{2}\right)+\frac{3 K}{2} z^{2} \\
& +\frac{a_{1}}{3}\left(x^{3}-3 x y^{2}\right)+\frac{a_{2}}{3} z^{3}+\frac{a_{4}}{4} z^{4}+\frac{a_{5}}{4}\left(x^{2}+y^{2}\right)^{2}
\end{aligned}
$$

where $G$ is the shear modulus, $K$ the bulk modulus and $a_{i}(i=1, \ldots, 5)$ are parameters of the model. By deriving the energy density (5) with respect to the strain tensor, we obtain 


$$
\begin{aligned}
\mathbf{S} & =z\left(3 K+a_{2} z+a_{4} z^{2}\right) \frac{\mathbf{I}}{\sqrt{3}}+\left[2 G+\frac{a_{5}}{2}\left(x^{2}+y^{2}\right)\right] \mathbf{E}_{d} \\
& +\sqrt{6} a_{1}\left[\left(\mathbf{E}_{d}\right)^{2}-\frac{x^{2}+y^{2}}{3} \mathbf{I}\right]
\end{aligned}
$$

\section{Local contact modeling}

For notational convenience, we assume that the contact with friction may occur between some points of two bodies A and B. The contact and friction laws are written in terms of relative velocity $\dot{\mathbf{u}}=\dot{\mathbf{u}}_{A}-\dot{\mathbf{u}}_{B}$ and of contact reactions $\mathbf{r}$. The following contact bi-potential is introduced by de Saxcé and Feng [5]:

$$
b_{c}(-\dot{\mathbf{u}}, \mathbf{r})=\bigcup_{\mathbb{R}_{-}}\left(-\dot{u}_{n}\right)+\bigcup_{K_{\mu}}(\mathbf{r})+\mu r_{n}\left\|-\dot{\mathbf{u}}_{t}\right\|
$$

where $\left.\left.\mathbb{R}_{-}=\right]-\infty, 0\right], K_{\mu}$ is the Coulomb's cone and $\bigcup$ stands for the indicator function. In order to avoid nondifferentiable potentials that occur in contact problems, it is convenient to use the Augmented Lagrangian Method [5, 15]. For the contact bi-potential $b_{c}$, we have:

$$
\forall \mathbf{r}^{\prime} \in K_{\mu}, \quad \varrho \mu\left(r_{n}^{\prime}-r_{n}\right)\left\|\dot{\mathbf{u}}_{t}\right\|+\left(\mathbf{r}^{\prime}-(\mathbf{r}-\varrho \dot{\mathbf{u}})\right)\left(\mathbf{r}^{\prime}-\mathbf{r}\right) \geq 0
$$

where $\varrho$ is a solution parameter which is not user-defined. The inequality (8) means that $\mathbf{r}$ is the projection of $\boldsymbol{\tau}$ onto the closed convex Coulomb's cone:

$$
\mathbf{r}=\operatorname{proj}\left(\boldsymbol{\tau}, K_{\mu}\right)
$$

For the numerical solution of the implicit equation (9), Uzawa's algorithm can be used, which leads to an iterative process involving one predictor-corrector step:

$$
\begin{aligned}
& \text { Predictor } \boldsymbol{\tau}^{i+1}=\mathbf{r}^{i}-\varrho^{i}\left(\dot{\mathbf{u}}_{t}^{i}+\left(\dot{u}_{n}^{i}+\mu\left\|\dot{\mathbf{u}}_{t}^{i}\right\|\right) \mathbf{n}\right) \\
& \text { Corrector } \mathbf{r}^{i+1}=\operatorname{proj}\left(\boldsymbol{\tau}^{i+1}, K_{\mu}\right)
\end{aligned}
$$

\section{Global time stepping}

Generally, mechanical behaviors of solids under contact/impact conditions are governed by a set of nonlinear equations

$$
\mathbf{M} \ddot{\mathbf{u}}=\mathbf{F}+\mathbf{R}, \quad \text { where } \mathbf{F}=\mathbf{F}_{\text {ext }}-\mathbf{F}_{\text {int }}-\mathbf{A} \dot{\mathbf{u}}
$$

where $\mathbf{M}$ is the mass matrix, $\mathbf{F}_{\text {ext }}$ the applied forces vector, $\mathbf{F}_{\text {int }}$ the internal forces vector and $\mathbf{R}$ the reaction forces vector. Taking the derivative of $\mathbf{F}_{\text {int }}$ with respect to the nodal displacements $\mathbf{u}$ gives the tangent stiffness matrix K. The most common method to integrate Eq.(11) is the Newmark 
method which is based on a second order algorithm. However, in impact problems, higher order approximation does not necessarily mean better accuracy, and may even be superfluous. At the moment of a sudden change of contact conditions (impact, release of contact), the velocity and acceleration are not continuous, and excessive regularity constraints may lead to serious errors. For this reason, Jean [16] has proposed a first order algorithm which is used in this work. This algorithm is based on the following approximations:

$$
\begin{gathered}
\int_{t}^{t+\Delta t} \mathbf{M} d \dot{\mathbf{u}}=\mathbf{M}\left(\dot{\mathbf{u}}^{t+\Delta t}-\dot{\mathbf{u}}^{t}\right) \\
\int_{t}^{t+\Delta t} \mathbf{F} d t=\Delta t\left((1-\xi) \mathbf{F}^{t}+\xi \mathbf{F}^{t+\Delta t}\right) \\
\int_{t}^{t+\Delta t} \mathbf{R} d t=\Delta t \mathbf{R}^{t+\Delta t} \\
\mathbf{u}^{t+\Delta t}-\mathbf{u}^{t}=\Delta t\left((1-\theta) \dot{\mathbf{u}}^{t}+\theta \dot{\mathbf{u}}^{t+\Delta t}\right)
\end{gathered}
$$

where $0 \leq \xi \leq 1 ; 0 \leq \theta \leq 1$. In the iterative solution procedure, all the values at time $t+\Delta t$ are replaced by the values of the current iteration $i+1$. Without going into details, we obtain the recursive form of (11) in terms of displacements:

$$
\begin{aligned}
\overline{\mathbf{K}}^{i} \Delta \mathbf{u} & =\overline{\mathbf{F}}^{i}+\overline{\mathbf{F}}_{a c c}^{i}+\mathbf{R}^{i+1} \\
\mathbf{u}^{i+1} & =\mathbf{u}^{i}+\Delta \mathbf{u}
\end{aligned}
$$

where the so-called effective terms are given by

$$
\begin{gathered}
\overline{\mathbf{K}}^{i}=\xi \mathbf{K}^{i}+\frac{1}{\theta \Delta t^{2}} \mathbf{M}^{i} \\
\overline{\mathbf{F}}_{a c c}^{i}=-\frac{1}{\theta \Delta t^{2}} \mathbf{M}^{i}\left\{\mathbf{u}^{i}-\mathbf{u}^{t}-\Delta t \dot{\mathbf{u}}^{t}\right\} \\
\overline{\mathbf{F}}^{i}=(1-\xi)\left(\mathbf{F}_{\text {int }}^{t}+\mathbf{F}_{\text {ext }}^{t}\right)+\xi\left(\mathbf{F}_{\text {int }}^{i}+\mathbf{F}_{\text {ext }}^{t+\Delta t}\right)
\end{gathered}
$$

\section{Numerical results}

The Bi-First algorithm presented above has been implemented and tested in the finite element code FER/Impact [17]. Due to the limitation of pages, we briefly present two examples of application.

The first example, proposed initially by Love and Laursen [18] who consider only linearly elastic materials, accounts for hyperelastic large deformations in the present work. The simulation consists of two three-dimensional blocks (Figure 1) that impact with relative tangential motion. The $L V F$ model is considered here with the initial shear modulus $G$ and bulk modulus $K$ same as in [18] (scaled units): $G=5000, K=3333$. Other parameters are: $a_{1}=50$, 
$a_{2}=50, a_{3}=0, a_{4}=2000$ and $a_{5}=100$. The total simulation time is 1 scaled time unit and the solution parameters are: $\Delta t=10^{-2}, \xi=\theta=0.5$. In order to investigate the frictional effects on the energy dissipation, different coefficients of Coulomb friction are used: $\mu=0.0,0.2,0.5,0.8$.

Figures 2,3 show the plots of the kinetic energy $E_{k}$ and the total energy $E_{t}$. We observe that the total energy is quite well conserved in the case of frictionless contact. However, in the case of frictional contact, the total energy decreases. So the total energy is dissipated by frictional effects as expected. It is worth noting that the dissipated energy is quantitatively calculated.

It is also interesting to examine another question: is the dissipated energy proportional to the friction coefficient? The answer is negative according to numerical results. The proof is illustrated by Figure 3 in which we observe almost the same dissipated energy even with two different friction coefficients $(\mu=0.2,0.5)$. In addition, the dissipated energy is less in the case $\mu=0.8$ than in the case $\mu=0.2$ or $\mu=0.5$. In fact, when the friction coefficient increases, the friction forces increase. However, the tangential slips will decrease. We know that the dissipated energy depends not only on the friction forces but also on the tangential slips on the contact surface.

Figure 4 shows the evolution of the von Mises stress at point A (see Figure 1). It can be seen that when the friction coefficient increases, the stress level becomes more important. The trajectory of point B in the plane BCD (see Figure 1 is depicted in Figure 5. We observe that the amplitude of the displacements increases with friction coefficient as expected.

The second example simulates the deformable multibody contact between Blatz-Ko hyperelastic bodies. In doing so, we wish to further explore the performance of the present method and the developed code FER/Impact in a large strain context and with complicated contact surfaces. In addition, this example would illustrate the possibility to investigate the heterogeneous behavior of granular materials involving both deformations of grains and the interaction of grains with friction. The problem is displayed in Figure 6. Several grains meshed with triangular elements are locked up in a rigid box. The left side of the box is given an horizontal motion so as to compress the grains. The contours of von Mises stress are depicted in Figure 7 from which we observe the effect of friction on the top and bottom surfaces. We observe also the stress concentrated zones as expected.

\section{Conclusion}

The main purpose of this paper is to briefly present the recent development of the bi-potential method applied to dynamic analysis of contact problems with Coulomb friction between hyperelastic bodies. Numerical results demonstrate that the Bi-First algorithm for local analysis of frictional contact problems and for global time integration of dynamics equations is suitable for a wide range of engineering applications. 


\section{References}

1. N. Kikuchi and J. T. Oden. Contact problems in elasticity: A study of variational inequalities and finite elements. Philadelphia: SIAM, 1988.

2. Z. H. Zhong. Finite element procedures in contact-impact problems. Oxford University Press, 1993.

3. P. Wriggers. Computational contact mechanics. John Wiley \& Sons, 2002.

4. T. A. Laursen. Computational contact and impact mechanics: Fundamentals of Modeling Interfacial Phenomena in Nonlinear Finite Element Analysis. Springer Verlag, 2002.

5. G. De Saxcé and Z.-Q. Feng. The bi-potential method: a constructive approach to design the complete contact law with friction and improved numerical algorithms. Mathematical and Computer Modeling, 28(4-8):225-245, 1998. Special issue: Recent Advances in Contact Mechanics.

6. Z.-Q. Feng. Some test examples of $2 \mathrm{D}$ and $3 \mathrm{D}$ contact problems involving coulomb friction and large slip. Mathematical and Computer Modeling, 28(48):469-477, 1998. Special issue: Recent Advances in Contact Mechanics.

7. Z.-Q. Feng, F. Peyraut, and N. Labed. Solution of large deformation contact problems with friction between Blatz-Ko hyperelastic bodies. Int. J. Engng. Science, 41:2213-2225, 2003.

8. H. M. Hilber, T. J. R. Hughes, and Taylor R. L. Improved numerical dissipation for the time integration algorithms in structural dynamics. Earthquake Engng. Struc. Dyn., 5:283-292, 1977.

9. M. Jean. The non-smooth contact dynamics method. Comp. Meth. Appl. Mech. Engng., 177:235-257, 1999.

10. Z.-Q. Feng, B. Magnain, J.-M. Cros, and P. Joli. Energy dissipation by friction in dynamic multibody contact problems. In Z.-H. Yao, M.-W. Yuan, and W.-X. Zhong, editors, Computational Mechanics, Beijing, China, Sept. 2004. WCCM VI in conjunction with APCOM04, Springer.

11. P.J. Blatz and W.L. Ko. Application of finite elastic theory to the deformation of rubbery materials. Transactions of the Society of Rheology, 6:223-251, 1962.

12. R.W. Ogden. Non-linear elastic deformations. Ellis Horwood, 1984.

13. A.N. Gent. A new constititive relation for rubber. Rubber Chem. Technol., 69:59-61, 1996.

14. E. Lainé, C. Vallée, and D. Fortuné. Nonlinear isotropic constitutive laws: choice of the three invariants, convex potentials and constitutive inequalities. Int. J. Engng. Science, 37:1927-1941, 1999.

15. J. C. Simo and T. A. Laursen. An augmented lagrangian treatment of contact problems involving friction. Computers \& Structures, 42:97-116, 1992.

16. M. Jean. Dynamics with partially elastic shocks and dry friction: double scale method and numerical approach. In 4th Meeting on unilateral problems in structural analysis, 1989. Capri.

17. Z.-Q. Feng. http://gmfe16.cemif.univ-evry.fr:8080/ feng/FerImpact.html.

18. G. R. Love and T. A. Laursen. Improved implicit integrators for transient impact problems: dynamic frictional dissipation within an admissible conserving framework. Comp. Meth. Appl. Mech. Engng., 192:2223-2248, 2003. 


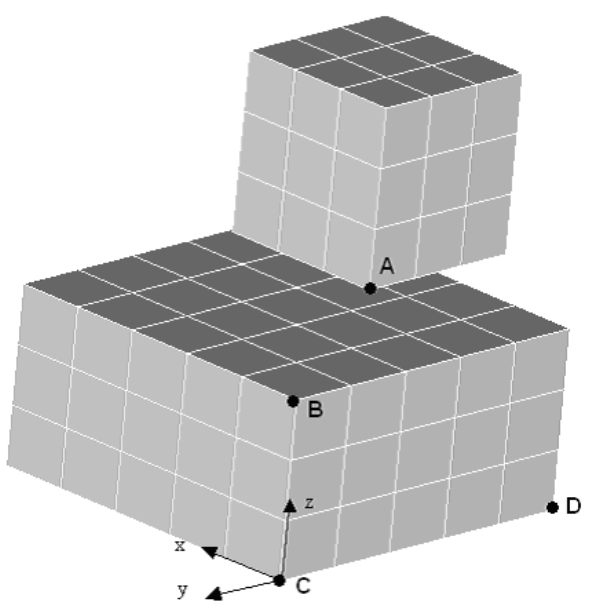

Fig. 1. Initial configurations and meshes

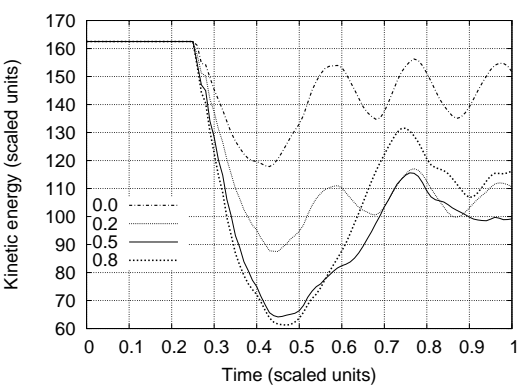

Fig. 2. Kinetic energy with different $\mu$

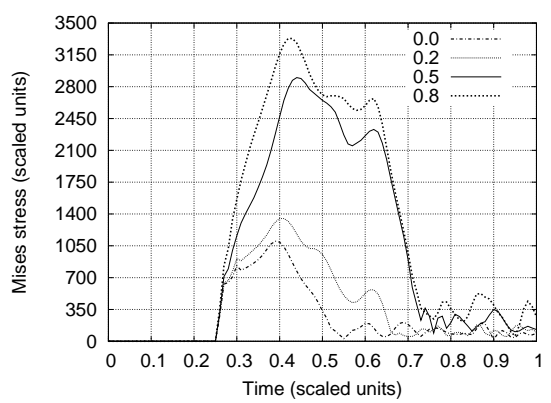

Fig. 4. von Mises stress at point A

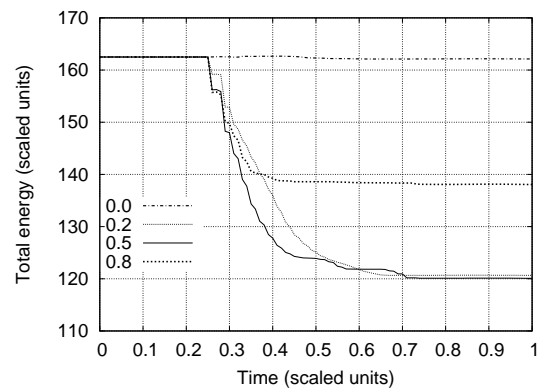

Fig. 3. Total energy with different $\mu$

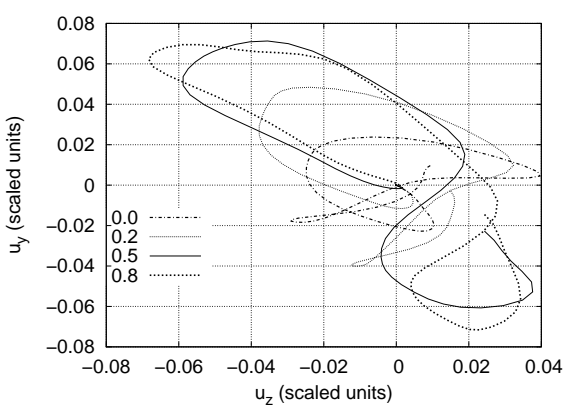

Fig. 5. Trace of point B 


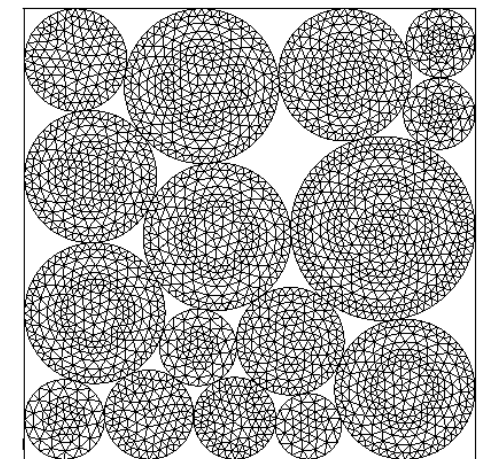

Fig. 6. Multibody contact: initial mesh

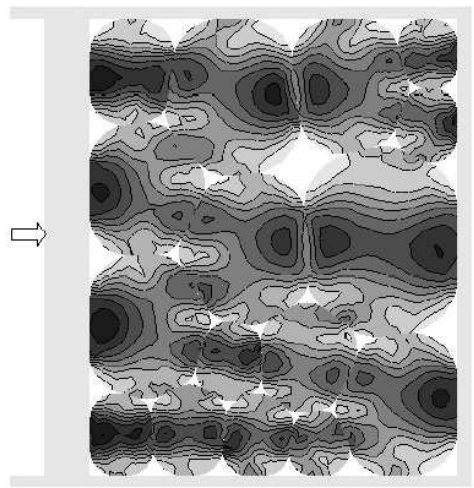

Fig. 7. Multibody contact: von Mises stress after loading 